\title{
Harmonic and supercontinuum generation in quadratic and cubic nonlinear optical media
}

\author{
S. Wabnitz* and V. V. Kozlov \\ Dipartimento di Ingegneria dell'Informazione, Università di Brescia, Via Branze 38, 25123 Brescia, Italy \\ *Corresponding author: stefano.wabnitz@ing.unibs.it
}

Received April 14, 2010; revised July 9, 2010; accepted July 13, 2010;

posted July 13, 2010 (Doc. ID 127036); published August 5, 2010

\begin{abstract}
We present numerical simulations by means of the generalized nonlinear envelope equation of harmonic and supercontinuum generation induced by femtosecond pump pulses in optical media with both quadratic and third-order nonlinearities. Application examples to higher harmonic generation in periodically poled lithium niobate and optical parametric generation of a mid-infrared continuum in gallium arsenide are discussed. (C) 2010 Optical Society of America

OCIS codes: $190.2620,190.5530,190.7110,320.6629,190.4410$.
\end{abstract}

\section{INTRODUCTION}

In recent years there has been a great deal of interest in research on high-order harmonic generation (HHG) [1] and supercontinuum (SC) [2] in nonlinear optical media for the control of light fields on time scales of the order of the optical carrier period. In particular, it has been shown that the generalized nonlinear envelope equation (GNEE) approach [3-5] is capable of modeling the sub-cycle dynamics in cubic nonlinear media with an accuracy which is comparable to the direct solution of Maxwell's equations [6]. In recent years efficient HHG has been predicted to occur in quadratic nonlinear media by means of numerical simulations [7], whereas SC [8-10] has been experimentally observed in both quadratic and cubic nonlinear media. However, theoretical modeling of these SC experiments has not yet been performed. Therefore interesting questions remain to be answered, for example, to which extent the observed SC generation is due to the quadratic or to the cubic (possibly through cascading effects) contributions to the nonlinear response of the material.

In this work we perform such a modeling based on the extension of the GNEE approach of Genty et al. [3] to the case of a non-centrosymmetric nonlinear medium with both second- and third-order contributions to the nonlinear polarization. This approach enables us to carry out numerical simulations that describe optical parametric amplification [11] or quasi-phase-matched second harmonic generation (SHG) in periodically poled crystals $[12,13]$ by means of a single equation for the total field envelope. Note that these well-known phenomena have been previously described in terms of separate equations for each of the interacting fields.

In Section 2 of this paper we present the theoretical derivation from the scalar wave equation of the GNEE equation for the field envelope (containing in principle arbitrarily fast temporal variations) subject to the effects of linear dispersion as well as both quadratic and cubic contributions to the nonlinear polarization. In Section 3 we validate the GNEE approach in the presence of a quadratic nonlinearity only and in the absence of linear dispersion, by comparing its solution with the direct solution of Maxwell's equations by means of the so-called pseudospectral spatial domain (PSSD) method [5]. We have considered here the rather extreme situation of a single cycle pulse. Finally, in Section 4 we present different examples of practical applications of the GNEE approach to describe frequency generation based on quasi-phasematching (QPM), whereby phase-matching between the fundamental harmonic and the second harmonic $(\mathrm{SH})$ is obtained by periodically poling the second-order nonlinear susceptibility $[12,13]$. First, we simulated higher harmonic and SC generation in periodically poled lithium niobate (PPLN) [9]. Next, we modeled the generation by optical parametric mixing of a mid-infrared (MIR) continuum in orientation-patterned gallium arsenide [10].

\section{EQUATIONS}

Let us consider the propagation of linearly polarized ultrashort pulses in a nonlinear medium exhibiting both quadratic and cubic nonlinearities, i.e., the total nonlinear polarization can be written as $P_{\mathrm{NL}}=P_{\mathrm{NL}}^{(2)}+P_{\mathrm{NL}}^{(3)}$ $=\varepsilon_{0}\left(\chi^{(2)} E^{2}+\chi^{(3)} E^{3}\right)$. In the frequency domain, the scalar wave equation reads as

$$
\left[\partial_{z}^{2}+k^{2}(\omega)\right] \widetilde{E}(z, \omega)=-\mu_{0} \omega^{2} \widetilde{P}_{\mathrm{NL}}(z, \omega)
$$

where

$$
\begin{gathered}
k^{2}(\omega)=\varepsilon(\omega) \omega^{2} / c^{2} \\
\widetilde{E}(z, \omega)=\int_{-\infty}^{+\infty} E(z, t) \exp (i \omega t) d t \\
\widetilde{P}_{\mathrm{NL}}(z, \omega)=\int_{-\infty}^{+\infty} P_{N L}(z, t) \exp (i \omega t) d t
\end{gathered}
$$




$$
\varepsilon(\omega)=(n(\omega)+i \alpha(\omega) c / 2 \omega)^{2}
$$

$n$ is the refractive index, and $\alpha$ is the linear loss coefficient. By using analytical methods such as the Green's function approach [14], one may transform Eq. (1) in the two coupled (via nonlinearity) first-order equations,

$$
\left[\partial_{z} \mp i k(\omega)\right] \widetilde{E}_{ \pm}(z, \omega)= \pm i \mu_{0} \frac{\omega^{2}}{2 k(\omega)} \widetilde{P}_{\mathrm{NL}}(z, \omega),
$$

for the forward, $\widetilde{E}_{+}(z, \omega)$, and backward, $\widetilde{E}_{-}(z, \omega)$, propagating components of the field $\widetilde{E}(z, \omega) \equiv \widetilde{E}_{+}(z, \omega)+\widetilde{E}_{-}(z, \omega)$.

Whenever the nonlinear polarization represents a relatively weak perturbation to the linear dielectric susceptibility, it is possible to neglect the coupling between counter-propagating waves [15-17]. This means that we may separately consider the first of Eqs. (2) that describes the evolution of the forward field component alone. By expressing this field as $E(z, t) \equiv\left(A(z, t) \exp \left(-i \omega_{0} t\right)+\right.$ c.c. $) / 2$ (dropping for simplicity the plus index), where $\omega_{0}$ and $A(z, t)$ are an arbitrary carrier frequency and an envelope function, one obtains

$$
\left[\partial_{z}-i k(\omega)\right] \widetilde{A}(z, \omega)=i \mu_{0} \frac{\omega^{2}}{2 k(\omega)} \widetilde{p}_{\mathrm{NL}}(z, \omega, \widetilde{A}),
$$

where

$$
\begin{gathered}
\tilde{A}(z, \omega)=\int_{-\infty}^{+\infty} A(z, t) \exp \left\{i\left(\omega-\omega_{0}\right) t\right\} d t \\
\widetilde{p}_{\mathrm{NL}}(z, \omega)=\int_{-\infty}^{+\infty} p_{\mathrm{NL}}(z, t) \exp \left\{i\left(\omega-\omega_{0}\right) t\right\} d t, \\
P_{\mathrm{NL}}(z, t) \equiv\left(p_{\mathrm{NL}}(z, t) \exp \left(-i \omega_{0} t\right)+\text { c.c. }\right) / 2,
\end{gathered}
$$

and $p_{\mathrm{NL}}=p_{\mathrm{NL}}^{(2)}+p_{\mathrm{NL}}^{(3)}$. Moreover,

$$
\begin{gathered}
p_{\mathrm{NL}}^{(2)}(z, t)=\varepsilon_{0} \chi^{(2)}\left(2|A|_{+}^{2} \exp \left(i \omega_{0} t\right)+A^{2} \exp \left(-i \omega_{0} t\right)\right) / 2, \\
p_{\mathrm{NL}}^{(3)}(z, t)=\varepsilon_{0} \chi^{(3)}\left(3|A|^{2} A+A^{3} \exp \left(-2 i \omega_{0} t\right)\right) / 4,
\end{gathered}
$$

where $|A|_{+}^{2}$ only contains frequency components with $\omega$ $\geq 0$. Therefore Eq. (3) can be written as

$$
\left[\partial_{z}-i \beta(\omega)+\frac{\alpha}{2}\right] \widetilde{A}(z, \omega)=i \rho(\omega) \widetilde{p}_{\mathrm{NL}}(z, \omega, \widetilde{A}),
$$

where $k(\omega)=n(\omega) \omega / c+\alpha(\omega) / 2 \equiv \beta(\omega)+\alpha(\omega) / 2$ and $\rho(\omega)$ $\equiv \omega / 2 n(\omega) c \varepsilon_{0}$, and we neglected the contribution of the loss coefficient $\alpha$ with respect to the propagation constant $\beta$ in the denominator of the right-hand side of Eq. (4). By performing a Taylor expansion of $\beta$ and $\rho$ around a carrier frequency $\omega_{0}$, one may transform Eq. (4) in the time domain and obtain the GNEE for the evolution of $A(z, t)$,

$$
\left[\partial_{z}-D+\frac{\alpha}{2}\right] A(z, t)=N p_{\mathrm{NL}}(z, t, A),
$$

where the dispersion operator $D$ is formally written as

$$
D=\sum_{m \geq 0} \frac{i^{m+1}}{m !}\left(\frac{\partial^{m} \beta}{\partial \omega^{m}}\right)_{\omega=\omega_{0}} \frac{\partial^{m}}{\partial t^{m}}
$$

and the nonlinearity operator $N$ is

$$
N=\sum_{m \geq 0} \frac{i^{m+1}}{m !}\left(\frac{\partial^{m} \rho}{\partial \omega^{m}}\right)_{\omega=\omega_{0}} \frac{\partial^{m}}{\partial t^{m}},
$$

where the series is typically truncated to the first-order term so that

$$
N \cong i \rho_{0}\left(1+i \tau_{s h} \partial / \partial t\right),
$$

where $\rho_{0} \equiv \omega_{0} / 2 n_{0} c \varepsilon_{0}, n_{0}=n\left(\omega_{0}\right)$, and

$$
\tau_{s h} \equiv 1 / \omega_{0}-\{\partial[\ln (n(\omega))] / \partial \omega\}_{\omega=\omega_{0}} .
$$

Equation (5) may be easily numerically solved by means of the standard split-step Fourier method, where the action of the dispersive step is computed as a phase shift in the frequency domain, and the nonlinear step is computed by a simple integration in the time domain. The strengths of the second- and third-order nonlinearities are typically measured in terms of $d_{\text {eff }}=\chi^{(2)} / 2$ and the nonlinear refractive index $n_{2}=3 \chi^{(3)} / 8 n_{0}$, respectively.

\section{MODEL VALIDATION}

In order to verify the accuracy of the GNEE (5), we compared its solution with the direct PSSD integration of Maxwell's equations [5]. For the sake of simplicity, we neglect dispersion and set $P \equiv P_{\mathrm{NL}}^{(2)}=\varepsilon_{0} \chi^{(2)} E^{2}$, with $\chi^{(2)}=0.02$. We consider the initial 1 cycle pulse $E(z=0, t)$ $=\cos \left(\omega_{p} t\right) \operatorname{sech}\left(0.3 \omega_{p} t\right)$ (with, e.g., $\lambda_{p}=2 \pi c / \omega_{p}=830 \mathrm{~nm}$ ).

Figure 1(a) compares the PSSD (solid curves) and GNEE (empty dots) solutions for the electric field after $6 \mu \mathrm{m}$, which indeed agrees with the prediction of Eq. (2) of [7]: this point corresponds to a carrier wave shock $[6,18,19]$, i.e., a vertical trailing edge for the central carrier oscillation; whereas Fig. 1(b) compares the associated spectral intensities showing $\mathrm{HHG}$ (of the carrier frequency $\left.\omega_{p}\right)$. As can be seen, there is a relatively good agreement between the solution of the GNEE (5) and the PSSD simulations. In all GNEE simulations, we only used $n=2^{11}$ sampling points in the physical spectral window, compared with at least $2^{14}$ data points for the PSSD

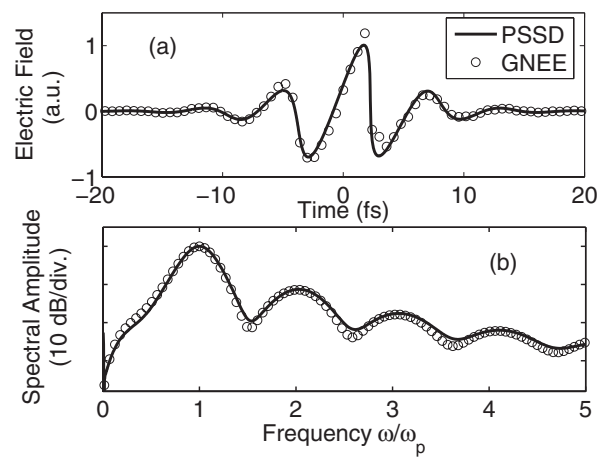

Fig. 1. Carrier wave shock induced by $\chi^{(2)}$ : comparison of PSSD (solid curves) and GNEE (empty dots) solutions for (a) the electric field and (b) the spectral amplitude of 1 cycle pulse at $830 \mathrm{~nm}$ at the carrier wave shock point as predicted by Eq. (2) of [7]. 
runs. For better numerical stability with a quadratic nonlinearity, we applied Oszag's $2 / 3$ rule by padding with $n / 3$ zeros the upper and lower parts of the spectral range. The GNEE simulation in Fig. 1 only took $100 \mathrm{~s}$ when running MATLAB on a laptop personal computer. Clearly, a main advantage of the GNEE approach is that it permits one to include in a straightforward manner the frequencydependent linear propagation constant $\beta(\omega)$ in the modeling of short pulse propagation.

\section{APPLICATION EXAMPLES}

As examples of practical application of the singleequation description of wave propagation in quadratic and cubic nonlinear materials which is provided by the GNEE (5), let us consider in this section the two main parametric mixing processes leading to either frequency doubling or frequency-difference generation in quasiphase-matched crystals. Indeed, recent experiments by Langrock et al. [9] demonstrated dramatic SHG and HHG in PPLN. Figure 2(a) shows the wavelength dependence of both the group delay and the group velocity dispersion $D$ for light propagating along the extraordinary axis in $\mathrm{LiNbO}_{3}$ [20]. In the solution of Eq. (5), we used a squarewave spatial modulation of the second-order nonlinear coefficient from $+d_{\text {eff }}$ to $-d_{\text {eff }}$ with a QPM period $D$, where $d_{\text {eff }}=25.2 \mathrm{pm} / \mathrm{V}$ and $n_{2}=5.3 \times 10^{-15} \mathrm{~cm}^{2} \mathrm{~W}^{-1}$, and we compared the propagation of a $32 \mathrm{fs} 2 \mathrm{GW} / \mathrm{cm}^{2}$ pump pulse at either $2.4 \mu \mathrm{m}$ (Fig. 3) or $1.58 \mu \mathrm{m}$ (Figs. 4 and 5).

Figure 3 illustrates the spectrogram, the amplitude of the field envelope, and the spectral intensity profile generated after $5.8 \mathrm{~mm}$ of PPLN from the input 4 cycle pulse at $2.4 \mu \mathrm{m}$, where the QPM period of $D=34.5 \mu \mathrm{m}$ was obtained from the dispersion curve in Fig. 2. The spectrogram in Fig. 3 reveals the details of the temporal structure of the HHG process: a few-cycle $\mathrm{SH}$ pulse is generated with the same intensity and similar duration as the pump, along with a time-compressed weaker thirdharmonic pulse, whereas the fourth harmonic presents a continuous-wave radiation peak. The formation of a fewcycle $\mathrm{SH}$ pulse is facilitated by the fact that the fundamental harmonic and the $\mathrm{SH}$ are located on opposite sides of the zero dispersion wavelength of $\lambda_{\mathrm{ZDW}}=1.98 \mu \mathrm{m}$ [see Fig. 2(a)], which leads to the reduced group-velocity mismatch (GVM)-induced delay of $60 \mathrm{fs} / \mathrm{mm}$. We have propa-

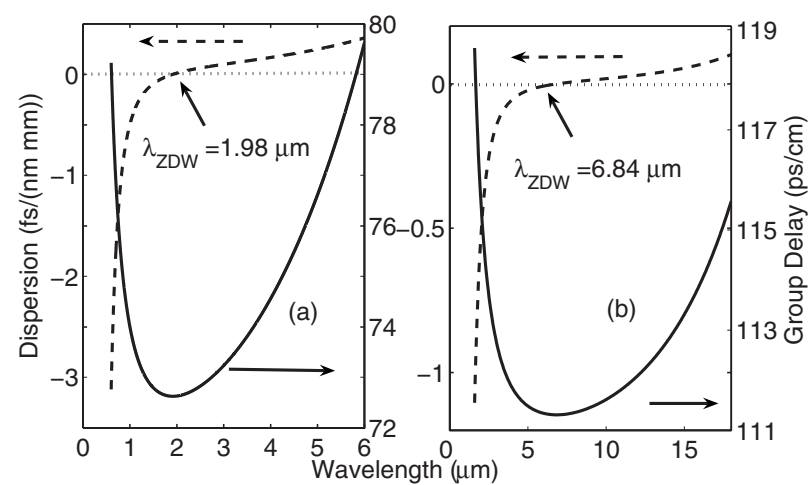

Fig. 2. Plot of dispersion and group delay versus wavelength for propagation along (a) the extraordinary axis of $\mathrm{LiNbO}_{3}$ or (b) in GaAs.

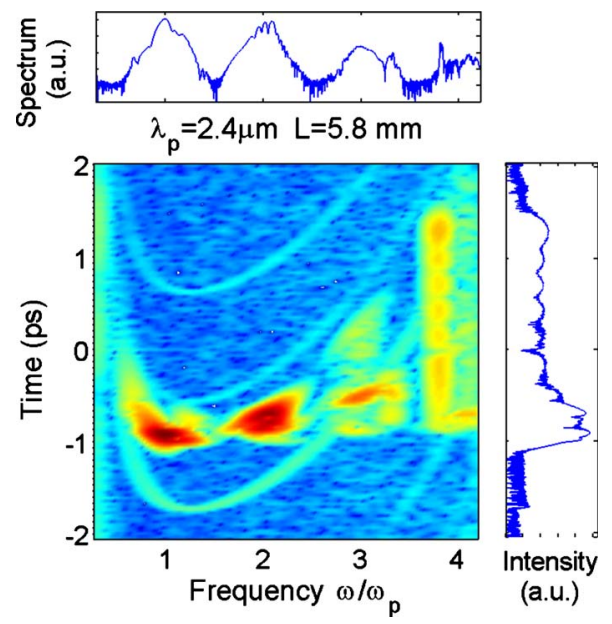

Fig. 3. (Color online) Display of the spectrogram (with a $25 \mathrm{fs}$ gate function), field envelope, and spectral amplitude profiles showing QPM-SHG of a $32 \mathrm{fs} 2 \mathrm{GW} / \mathrm{cm}^{2}$ pump pulse at $2.4 \mu \mathrm{m}$ after $5.8 \mathrm{~mm}$ of PPLN. The QPM period is $34.5 \mu \mathrm{m}$.

gated the field up to $17.5 \mathrm{~mm}$ and observed a total $500 \mathrm{fs}$ temporal walk-off between the fundamental and the $\mathrm{SH}$ pulses, which is half the value of that predicted from Fig. 2(a). The discrepancy results from the nonlinear trapping of the pump and $\mathrm{SH}$ pulses over the first $8 \mathrm{~mm}$ of propagation.

On the other hand, Figs. 4 and 5 show that for an input pump pulse at $1.58 \mu \mathrm{m}$ the GVM is so large that no mutual trapping with the $\mathrm{SH}$ is possible: as a result, the $\mathrm{SH}$ (and third-harmonic) energy is uniformly distributed in relatively long pulses. In the simulation in Fig. 4 the pump pulse duration and intensity were the same as in Fig. 3. Moreover, we kept the QPM period unchanged at $34.5 \mu \mathrm{m}$ as in Fig. 3 so that no phase-matching occurs between the pump and its SH. Still, it is quite remarkable to note that broadband higher harmonic generation is observed even for a strongly mismatched situation, albeit with a largely reduced peak efficiency. Clearly (as shown in Fig. 5) the SHG conversion efficiency is substantially improved whenever the QPM period is reduced to

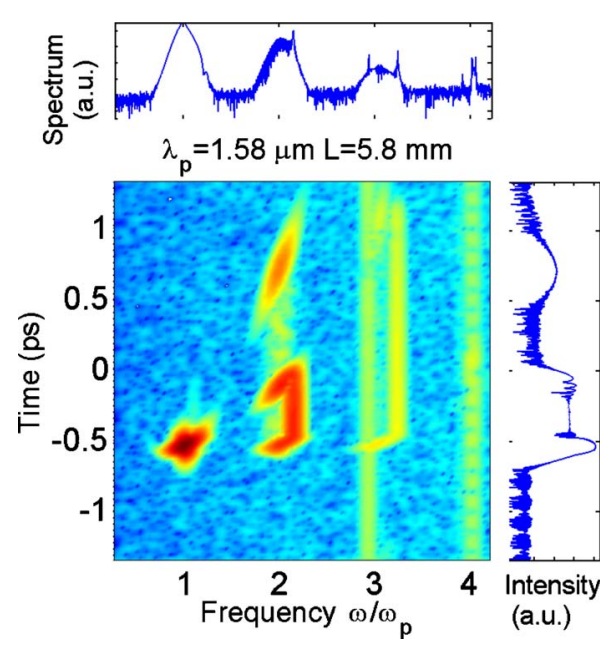

Fig. 4. (Color online) Same as Fig. 3, for pump pulse at $1.58 \mu \mathrm{m}$. 

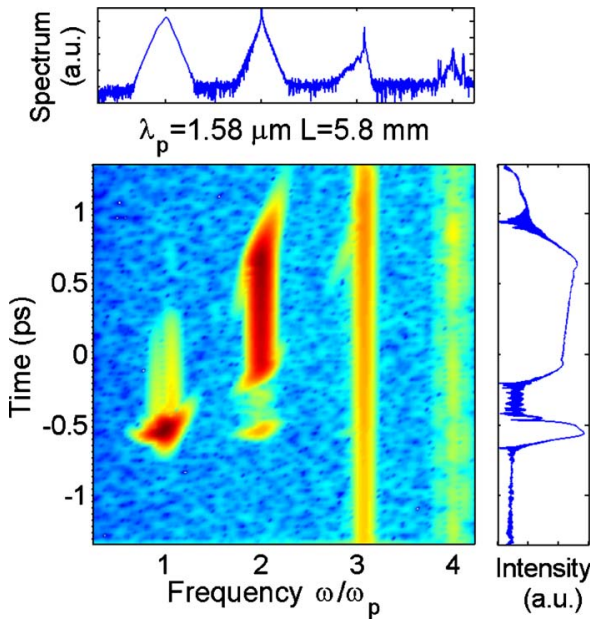

Fig. 5. (Color online) Same as Fig. 4, with the QPM period of $19.9 \mu \mathrm{m}$ that is adapted for SHG with a pump pulse at $1.58 \mu \mathrm{m}$.

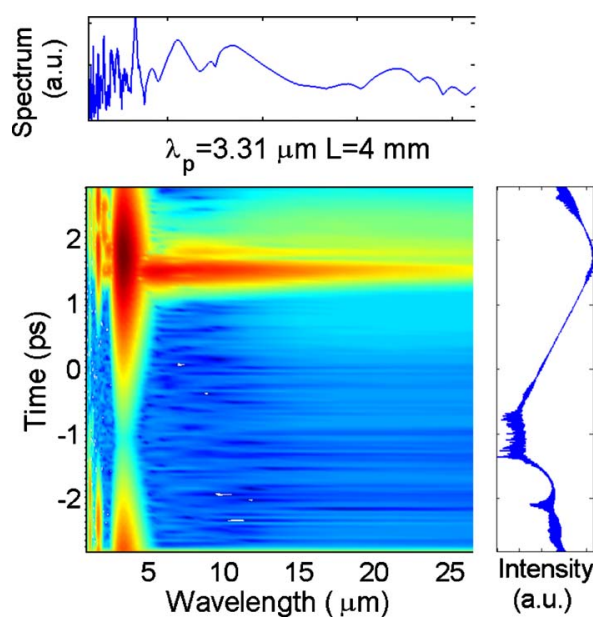

Fig. 6. (Color online) Same as Fig. 3, showing QPM-induced SC from the mixing of a $500 \mathrm{fs} 1 \mathrm{GW} / \mathrm{cm}^{2}$ pump at $3.31 \mu \mathrm{m}$ and a $100 \mathrm{fs} 20 \mathrm{MW} / \mathrm{cm}^{2}$ signal at $5.5 \mu \mathrm{m}$ in $4 \mathrm{~mm}$ of GaAs.

$19.9 \mu \mathrm{m}$ so that the pump at $1.58 \mu \mathrm{m}$ and its $\mathrm{SH}$ wavelength are now phase-matched.

Next we modeled by means of Eq. (5) the recently observed optical parametric generation of a MIR continuum in orientation-patterned GaAs [10], whose dispersion is illustrated in Fig. 2(b) [20]. Figure 6 shows the MIR SC extending from 5 to $10 \mu \mathrm{m}$ that results from the mixing of a $500 \mathrm{fs} 1 \mathrm{GW} / \mathrm{cm}^{2}$ pump pulse centered at $3.31 \mu \mathrm{m}$ with a $100 \mathrm{fs} 20 \mathrm{MW} / \mathrm{cm}^{2}$ signal pulse at $5.5 \mu \mathrm{m}$, after propagation through $4 \mathrm{~mm}$ of QPM-GaAs with $d_{\text {eff }}=69 \mathrm{pm} / \mathrm{V}, n_{2}$ $=1.5 \times 10^{-13} \mathrm{~cm}^{2} \mathrm{~W}^{-1}[10]$, and the QPM period of $163 \mu \mathrm{m}$. As shown in Fig. 2(b), in GaAs the signal at $5.5 \mu \mathrm{m}$ and the parametrically generated idler at $9.5 \mu \mathrm{m}$ are on opposite sides of the zero dispersion wavelength $\lambda_{\mathrm{ZDW}}=6.84 \mu \mathrm{m}$, and the resulting broadband GVM matching leads to wideband SC generation.

\section{CONCLUSIONS}

In conclusion we derived, validated, and provided application examples of an effective approach based on a single
GNEE describing the evolution of the arbitrarily fast optical field envelope. This method enables the efficient numerical study of ultrashort pulse propagation and frequency conversion in both quadratic and cubic nonlinear optical media.

\section{ACKNOWLEDGMENTS}

We acknowledge enlightening discussions with D. Modotto, M. Conforti, B. Kibler, and J. M. Dudley. This work was carried out with support from the Fondazione Cariplo grant No. 2009-2730.

\section{REFERENCES}

1. F. Krausz and M. Ivanov, "Attosecond physics," Rev. Mod. Phys. 81, 163-234 (2009).

2. J. M. Dudley, G. Genty, and S. Coen, "Supercontinuum generation in photonic crystal fiber," Rev. Mod. Phys. 78, 11351184 (2006)

3. G. Genty, P. Kinsler, B. Kibler, and J. M. Dudley, "Nonlinear envelope equation modelling of sub-cycle dynamics and harmonic generation in nonlinear waveguides," Opt. Express 15, 5382-5387 (2007).

4. G. Genty, B. Kibler, P. Kinsler, and J. M. Dudley, "Harmonic extended supercontinuum generation and carrier envelope phase dependent spectral broadening in silica nanowires," Opt. Express 16, 10886-10893 (2008).

5. P. Kinsler, "Optical pulse propagation with minimal approximations," Phys. Rev. 81, 013819 (2010).

6. J. C. A. Tyrrell, P. Kinsler, and G. H. C. New, "Pseudospectral spatial-domain: a new method for nonlinear pulse propagation in the few-cycle regime with arbitrary dispersion," J. Mod. Opt. 52, 973-986 (2005).

7. S. B. P. Radnor, L. E. Chipperfield, P. Kinsler, and G. H. C. New, "Carrier-wave steepened pulses and gradient-gated high-order harmonic generation," Phys. Rev. A 77, 033806 (2008).

8. N. K. M. Naga Srinavas, S. Sree Harsha, and D. N. Rao, "Femtosecond supercontinuum generation in a quadratic nonlinear medium (KDP)," Opt. Express 13, 3224-3229 (2005).

9. C. Langrock, M. M. Fejer, I. Hartl, and M. E. Fernmann, "Generation of octave-spanning spectra inside reverseproton-exchanged periodically poled lithium niobate waveguides," Opt. Lett. 32, 2478-2480 (2007).

10. P. S. Kuo, K. L. Vodopyanov, M. M. Fejer, D. M. Simanovskii, X. Yu, J. S. Harris, B. Bliss, and D. Weyburne, "Optical parametric generation of a mid-infrared continuum in orientation-patterned GaAs," Opt. Lett. 31, 71-73 (2006).

11. R. A. Baumgartner and R. L. Byer, "Optical parametric amplification," IEEE J. Quantum Electron. 15, 432-444 (1979).

12. M. M. Fejer, G. A. Magel, D. H. Jundt, and R. L. Byer, "Quasi-phase matched second harmonic generation: tuning and tolerances," IEEE J. Quantum Electron. 28, 2631-2654 (1992).

13. D. S. Hum and M. M. Fejer, "Quasi-phasematching," C. R. Phys. 8, 180-198 (2007).

14. A. Ferrando, M. Zacares, P. F. de Cordoba, D. Binosi, and A. Montero, "Forward-backward equations for nonlinear propagation in axially invariant optical systems," Phys. Rev. E 71, 016601 (2005)

15. A. I. Maimistov and A. M. Basharov, Nonlinear Optical Waves (Kluwer, 1999), Chap. 4, p. 137.

16. R. K. Bullough, P. M. Jack, P. W. Kitchenside, and R. Saunders, "Solitons in laser physics," Phys. Scr. 20, 364-381 (1979).

17. J. C. Eilbeck and R. K. Bullough, "The method of character- 
istics in the theory of resonant or nonresonant nonlinear optics," J. Phys. A 5, 820-829 (1972).

18. G. Rosen, "Electromagnetic shocks and the selfannihilation of intense linearly polarized radiation in an ideal dielectric material," Phys. Rev. 139, A539-A543 (1965)
19. R. G. Flesch, A. Pushkarev, and J. V. Moloney, "Carrier wave shocking of femtosecond optical pulses," Phys. Rev. Lett. 76, 2488-2491 (1996).

20. Dispersion data taken from Optical Society of America, Michael Bass, ed., Handbook of Optics, 2nd ed. (McGrawHill, 1994), Vol. 2. 